\title{
NEEDS OF ADULTS EDUCATION STAKEHOLDERS
}

\section{RELAZIONE / REPORT}

Aleksander Kobylarek, Luba Jakubowska, Kamil Błaszczyński, Noémie Govindin, Piera Sciama, Alcidio Jesus, Claudia Amaral, Samuel Yosef, Amelia Fiorillo 


\section{NEEDS OF ADULTS EDUCATION}

STAKEHOLDERS

\section{RELAZIONE / REPORT}

Aleksander Kobylarek, Luba Jakubowska, Kamil Błaszczyński, Noémie Govindin, Piera Sciama, Alcidio Jesus, Claudia Amaral, Samuel Yosef, Amelia Fiorillo 
The publication prepared as a result of The Erasmus+ Strategic Partnership Project in the Field of Adult Education titled Needs of adults education stakeholders ( $\mathrm{N}$ 2019-1-PLo1-KA204-065792) with experts from the consortium organizations: FUNDACIA PRO SCIENTIA PUBL CA (Poland), ITAKA TRAINING (Italy), E-SENIORS: INTTATION DES SENIORS AUX NTIC ASSOCATTON (France) and AFN ACA DEMIA FORMAÇ̄̃o Do NORTE (Portugal).

The European Commission's support for the production of this publication does not constitute an endorsement of the contents, which reflect the views only of the authors, and the Commission cannot be held responsible for any use which may be made of the information contained therein.

AUTHORS:

Aleksander Kobylarek, Luba Jakubowska, Kamil Błaszczyński, Noémie Govindin, Piera Sciama, Alcidio Jesus, Claudia Amaral, Samuel Yosef, Amelia Fiorillo

STATISTICAL EDITORS:

Kamil Btaszzcyński, University of Wroclaw, Poland

\section{REVIEWER}

Tijana Milenkovic Jankovic, the uK

TRANSLATION INTO ITALIAN AND PROOFREADING:

Samuel Yosef, Italy

DESIGN AND DTR:

Hanna Włoch, Poland

Foundation Pro Scientia Publica

U1. Redycka 37, 51-169 Wroctaw

www.proscientiapublica.pl
Il sostegno della Commissione europea alla produzione di questa pubblicazione non costituisce un'approvazione del contenuto, che riflette esclusivamente il punto di vista degli autori, e la Commissione non può essere ritenuta responsabile per l'uso che può essere fatto delle informazioni ivi contenute.

Febbraio 2021

Creative Commons Attribution 4.0 Internationa Authority code: CC_BY_4__o

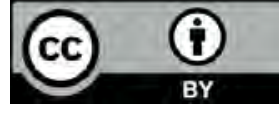

ISBN 978-83-953451-7-3

doi: 10.15503/andr.2021.neon.it

Photo by

CoWomen ; Green Chameleon ; krakenimages

Marten Bjork ; Sarah Dorweiler ; You X Ventu

on Unsplash
Introduzione 4

\section{Risultati 10}

Sommario 24

References 30

Annexes 34

Annex 136

Annex $11 \quad 40$

INDICE 
1. Introduzione 
Il progetto "Needs of adults education stakeholders" ( $\mathrm{N}^{\circ}$ 2019-1-PLo1-KA204-065792) è stato creato in risposta alla mancanza di uno strumento che misurasse i bisogni eduzione degli adulti.

Nonostante il vigoroso sviluppo della metodologia andragogica, mancano ancora deg strumenti di ricerca tipici per questo campo. L'andragogia utilizza strumenti di campi correlati, principalmente dalla psicologia Una tale soluzione non è soddisfacente, in primo luogo, perché molti strumenti hann un accesso limitato (principalmente dovuto alla necessità di pagare le tasse per l'utilizzo e l'obbligo per uno psicologo di essere presente nel team), e in secondo luogo, non sono specifici per studiare le esigenze delle organizzazioni per l'educazione degli adulti e le esigenze educative dei loro dipendenti.

In risposta al bisogno di cui sopra, i coordinatori del progetto hanno sviluppato un questionario per verificare tali esigenze. Il sondaggio è stato tradotto in altre lingue e condotto anche dai partner del progetto. Sulla base dell'analisi del contenuto delle risposte raccolte, è stata creata una classificazione dei bisogni educativi del personale di AE (Aleksander Kobylarek, Luba Jakubowska, Noémie Govindin, Piera Sciama, Alcidio Jesus, Claudia Amaral, Samuel Yosef, Amelia Fiorillo).

Il rapporto presentato contiene informazioni sui bisogni educativi e sulla valutazione quantitativa delle organizzazioni di educatori per adulti e lavoratori delle ONG nei paesi de consorzio (Polonia, Francia, Italia e Portogallo) e in altri paesi dell'UE e alcuni non UE. La ricerca ha visto la partecipazione di 1176 partecipanti. Ciò che è importante, è il fatto che la ricerca sia stata condotta in diversi paesi, non solo nei paesi del consorzio, elemento che garantisce sintomatici per il settore AE europeo.
STRUMENTI PER DIAGNOSTICARE

LE COMPETENZE CHIAVE

E LE ESIGENZE DI CAMBIAMENTO

NELL'ORGANIZZAZIONE

Per diagnosticare i bisogni educativi, è stato utilizzato lo strumento standardizzato dell'in-
ventario dei bisogni educativi degli adulti ventario dei bisogni educativi degli adulti
(AENI). Il questionario è uno strumento progettato per studiare le competenze chiave per i lavoratori dell'educazione degli adulti (Consiglio ropea, 2018). Il modello delle competenze è inscritto nel concetto di apprendimento permanente e non si concentra sulla funzione di compensazione del processo educativo; invece, promuove l'intuizione dell'educatore nello sviluppo personale e la sua partecipazione cosciente e attiva ad esso AfNi ̀̀ stato utilizzando le raccomandazioni dell'Ue sulle competenze chiave del XXI secolo. Sulla base di queste raccomandazioni, il team di ricerca ha creato, testato e valutato AENI utilizzando metodi di analis statistica. L'affidabilità del test è stata confermata attraverso l'intercorrelazione. L'affidabilità del questionario è stata confermata attraverso l'analisi fattoriale. Inoltre, l'affidabilità teorica è stata verificata attraverso la correlazione dell'AENI con il Profilo delle Competenze Sociali (PROKOS) (Martowska, Matczak, 2013), che misura le competenze social nelle seg nelle segrentiare: assetivita, cooperazione, mentalità sociale, intraprendenza e consapevolezza della comunità. Al fine di indagare l'attendibilità teorica, sono state formulate ipotesi sia sulla correlazione dei punteggi generali (dai questionari AENI e PROKOS) si sulle scale selezionate. Studi di correlazione, a cui hanno partecipato 98 intervistati, hanno confermato l'affidabilità teorica del questionario AENI.

Prima della ricerca finale, è stata condotta un'analisi discriminante sotto forma di test di affidabilità Alpha-Cronbach (c di afficiabi partecipanti) che ha rivelato un'altissima affidabilità dello strumento creato $(\alpha \mathrm{di}$ Cronbach $=0,846)$ e le sue dimensioni.

L'AENI ha fornito due informazioni principali: punteggio generale di un individuo e punteggi in sei sottocategorie diagnostiche: 1) competenze comunicative (cc);

2) competenze multilingue e multicultural (ML\&MC);

3) competenze digitali (DC);

4) competenze imprenditoriali (EC);

5) apertura per la scienza e la cultura (OSC);

6) competenze sociali e civili (scc).
Tutte le sottocategorie sono state create dal team di ricerca polacco (Kobylarek, Jakubowska, Błaszczyński 2020 - non ancora pubblicato ufficialmente), giudici competenti e utilizzando strumenti statistici come 1) anlisi fattorian ) analisi fattoriale principle fidabilità alfa di Cronbach e 3) Kendall W test di associazione. Ogni individuo può ottenere un massimo del $100 \%$ dal test in generale e dai punteggi delle sottocategorie. Più il punteggio più vicino doveva arrivare al 100\%, meno visibili erano i bisogn educativi dell'individuo.

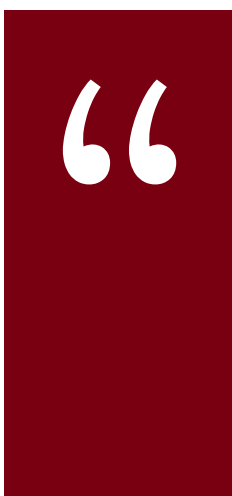

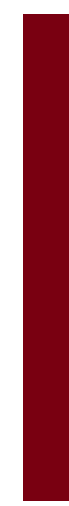

Per questo rapporto si suggerisce di utilizzare la classificazione dei punteggi come segue:

\section{0-44\% - punteggio basso - bisogni educativi medio}

45-75\% - mediocri - bisogni educativi medio

76-10o\% - alto - bisogni educativi debolmente sentiti
Per dare agli intervistati la possibilità di valutare la propria organizzazione, è stat condotto un sondaggio di 18 domande. L'indagine era composta da 18 affermazioni che rappresentano 18 categorie (Tabella 1) a cui agli intervistati è stato chiesto di contrassegnare il loro rispettivo atteggiamento su una scala di 5 punti che va da «decisamente non d'accordo» a «decisamente d'accordo».
Gli elementi inclusi nel sondaggio sono stati creati sulla base della teoria a due fattori della motivazione al lavoro di Herzberg (fonte: Bassett-Jones, Lloyd, 2005). I puntegoi erano rappresentati in valori \%. Più alto è il punteggio ottenuto da un individuo, migliore è stata la sua opinione sulla sua organizzazione e minore è la necessità di cambiamento nell'organizzazione. 
Tabella 1. Voci di valutazione dell’organizzazione - Elenco e descrizione

\begin{tabular}{|c|c|}
\hline $\begin{array}{l}\text { Apprezzamento da parte del } \\
\text { datore di lavoro }\end{array}$ & Il dipendente si sente apprezzato dal suo datore di lavoro. \\
\hline $\begin{array}{l}\text { Apprezzamento da parte del } \\
\text { leader }\end{array}$ & $\begin{array}{l}\text { Il dipendente si sente apprezzato dal suo diretto leader } \\
\text { o supervisore. }\end{array}$ \\
\hline $\begin{array}{l}\text { Raggiungimento degli obiettivi } \\
\text { per l'organizzazione }\end{array}$ & $\begin{array}{l}\text { Il dipendente può raggiungere gli obiettivi fissati dal suo } \\
\text { datore di lavoro. }\end{array}$ \\
\hline $\begin{array}{l}\text { Raggiungimento del successo } \\
\text { personale }\end{array}$ & $\begin{array}{l}\text { Il dipendente ha l'opportunità di raggiungere i suoi succes- } \\
\text { si personali nell'organizzazione. }\end{array}$ \\
\hline Responsabilità & $\begin{array}{l}\text { Il dipendente si sente fortemente responsabile per i compi- } \\
\text { ti che sta conducendo. }\end{array}$ \\
\hline Indipendenza & $\begin{array}{l}\text { Il dipendente ha una vasta gamma di libertà nel raggiungi- } \\
\text { mento del compito commissionato. }\end{array}$ \\
\hline Avanzamento & $\begin{array}{l}\text { Il datore di lavoro offre ai dipendenti possibilità di avanza- } \\
\text { mento. }\end{array}$ \\
\hline Crescita personale & $\begin{array}{l}\text { Il lavoro attuale influisce fortemente positivamente sullo } \\
\text { sviluppo personale dei dipendenti. }\end{array}$ \\
\hline $\begin{array}{l}\text { Strategia di sviluppo } \\
\text { dell’organizzazione }\end{array}$ & $\begin{array}{l}\text { L'organizzazione del dipendente ha una chiara strategia di } \\
\text { sviluppo e il dipendente la comprende. }\end{array}$ \\
\hline $\begin{array}{l}\text { Continuità dello sviluppo } \\
\text { dell'organizzazione }\end{array}$ & $\begin{array}{l}\text { Lorganizzazione non ferma i tentativi di ulteriore miglio- } \\
\text { ramento e si sforza continuamente di perfezionare ed es- } \\
\text { pandere la propria attività. }\end{array}$ \\
\hline Posto di lavoro & $\begin{array}{l}\text { L'organizzazione fornisce ai dipendenti un posto di lavoro } \\
\text { adeguato alle attività commissionate. }\end{array}$ \\
\hline $\begin{array}{l}\text { Comfort del lavoro nell'organiz- } \\
\text { zazione }\end{array}$ & $\begin{array}{l}\text { L'organizzazione pone l'accento sul comfort dei dipenden- } \\
\text { ti sul lavoro. }\end{array}$ \\
\hline Stipendio & $\begin{array}{l}\text { Il dipendente è soddisfatto del suo stipendio guadagnato } \\
\text { nellorganizzazione. }\end{array}$ \\
\hline Vantaggi e bonus & $\begin{array}{l}\text { L'organizzazione mette a disposizione dei dipendenti una } \\
\text { ricca offerta di vantaggi e bonus supplementari. }\end{array}$ \\
\hline Rapporti con Boss & $\begin{array}{l}\text { Il dipendente ha buoni rapporti personali con il suo datore } \\
\text { di lavoro. }\end{array}$ \\
\hline $\begin{array}{l}\text { Rapporti con i colleghi nell'or- } \\
\text { ganizzazione }\end{array}$ & $\begin{array}{l}\text { Il dipendente ha buoni rapporti personali con i suoi colle- } \\
\text { ghi di lavoro. }\end{array}$ \\
\hline Sicurezza sul lavoro & $\begin{array}{l}\text { L'organizzazione è finanziariamente stabile e dà ai dipen- } \\
\text { denti una sensazione di stabilità occupazionale. }\end{array}$ \\
\hline Lavoro e vita privata & $\begin{array}{l}\text { Le attività svolte nell'organizzazione influiscono negativa- } \\
\text { mente sulla vita privata del dipendente. }\end{array}$ \\
\hline
\end{tabular}

(fonte: Gli autori proprio sulla base di:Herzberg, Mausner, Snyderman, 1959)
INTERPRETAZIONE DEI RISULTATI

DELLO STUDIO SULLA NECESSITÀ

DI CAMBIAMENTO NELLE

ORGANIZZAZIONI

Ogni elemento può avere un punteggio minimo dello $0 \%$ e un punteggio massimo del $100 \%$. Più alto era il punteggio ottenuto dall'individuo, migliore era la sua opinione sulla sua organizzazione. L'unica eccezione era l'ultima categoria incentrata sul lavoro e sulla vita privata, in questa categoria più più debole ra l'influenza del lavoro sulla propria vita privata, quindi migliore era il punteggio.

Si raccomanda di interpretare i punteggi ottenuti riguardo alla valutazione dell'organizzazione secondo la classificazione proposta di seguito:

$\mathbf{0 - 2 0} \%$ - molto bassa - pessima opinione sull’organizzazione / datore di lavoro

21-40\% - bassa - cattiva opinione sull'organizzazione / datore di lavoro

41-6o\% - media - opinione neutra sullorganizzazione / datore di lavoro

61-80\% - alto - buona opinione sull’organizzazione / datore di lavoro

81-100\% - molto alto - ottima opinione sull'organizzazione / datore di lavoro

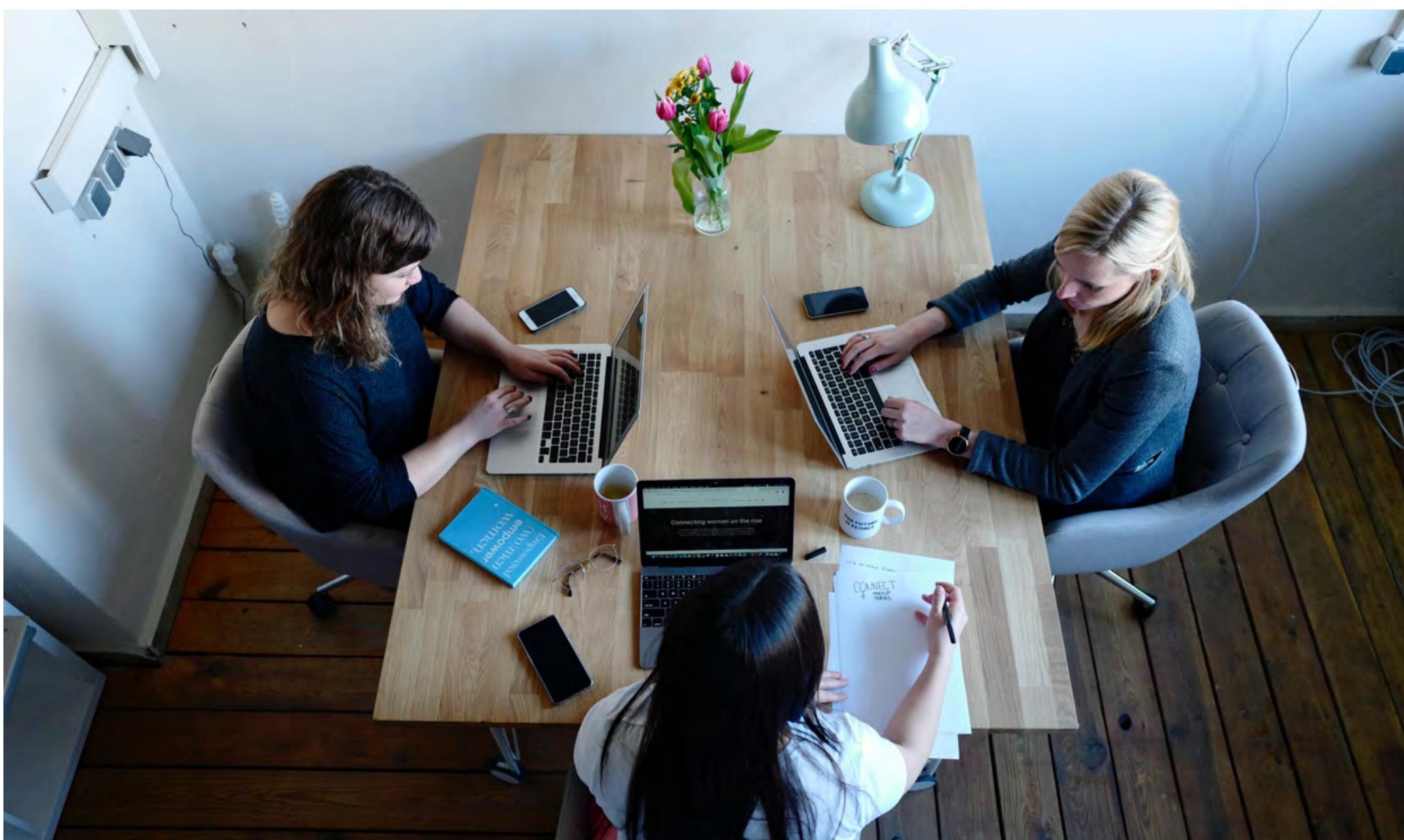


2. Risultati

\section{RISULTATI}

wet? 
RISULTATI GENERALI PER LE

RISPETTIVE COMPETENZE NEL

TEST AENI

I risultati della diagnosi condotta sono stat suddivisi in tre livelli di analisi: 1) puntegg generali ottenuti da tutti i partecipanti allo studio; 2) confronto dei punteggi ottenuti dai partecipanti che erano cittadini formali di un paese dell'uE rispetto ai partecipant che erano cittadini formali di paesi non UE; 3) confronto dei puntegi ottenuti dai pinipali partecipanti al progetto quindi: Francia,

Figure 1. Test dell'inventario per l'istruzione degli adulti

Punteggi medi delle sottocategorie

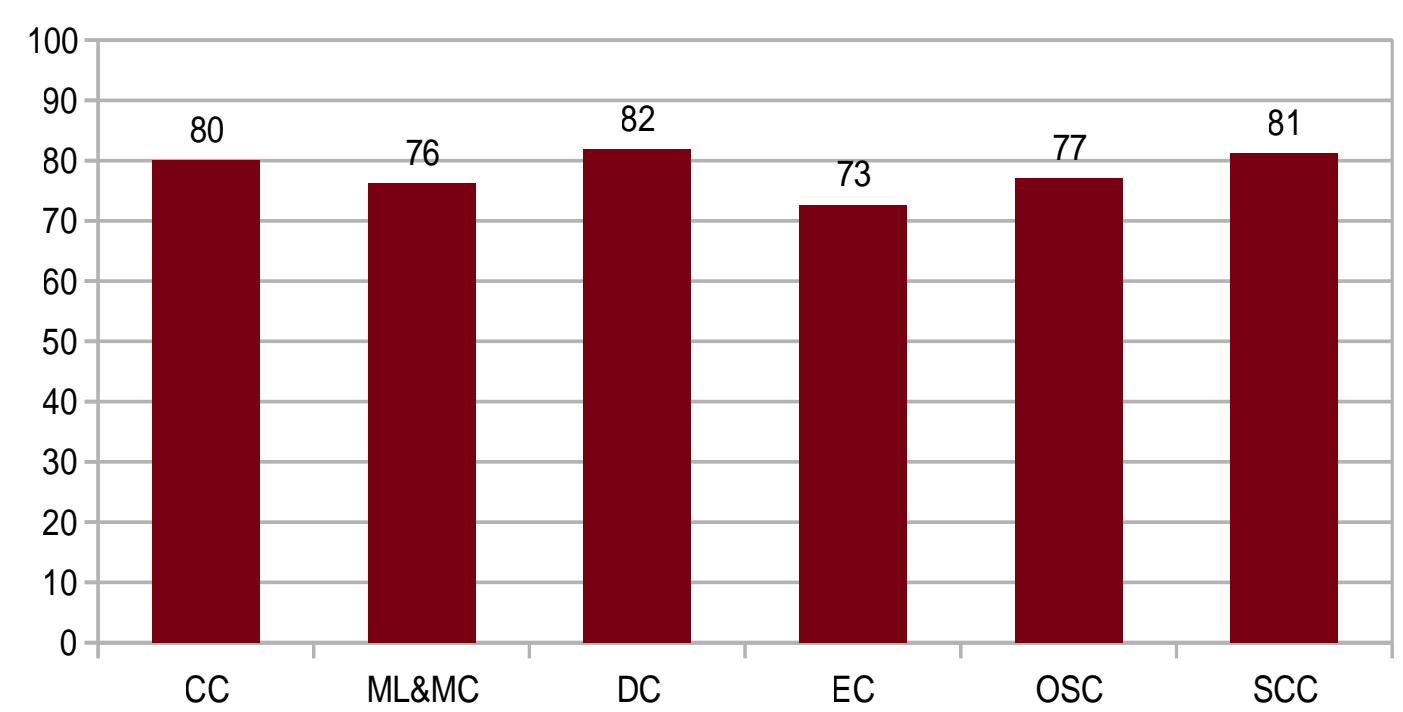

DIAGNOSI DELLE ESIGENZE

DI CAMBIAMENTO NELLE

ORGANIZZAZIONI

I risultati ottenuti nella valutazione del sondaggio sull'organizzazione avevano rivelato un punteggio medio generale del $69 \%$, che può essere considerato alto. L'analisi delle singole categorie ha rivelato che il punteg-
Italia, Polonia e Portogallo. Allo studio hanno partecipato in 1176, il cui punteggio generale medio dal test AENI era del $78 \%$, che può essere classificato come buono. Un'analisi dettagliata delle sottocategorie AEN ha rivelato che il punteggio più basso è stato ottenuto nelle competenze imprenditoriali $(73 \%)$ che possono essere classificate come buone. E i punteggi più alti sono stati rilevati nelle competenze digitali (82\%) e nelle competenze sociali e civili ( $81 \%)$, che possono essere considerate molto buone.
Figure 2. Valutazione del sondaggio sull organizzazione Punteggio medio delle sottocategorie

$\begin{array}{llllllllll}10 & 20 & 30 & 40 & 50 & 60 & 70 & 80 & 90 & 100\end{array}$

tilavoro

Apprezzamento da parte del leader

Raggiungimento degli obiettivi per l'organizzazione

Raggiungimento del successo personale

Responsabilità

Indipendenza

Avanzamento

Crescita personale

Strategia di sviluppo dell'organizzazione

Continuità dello sviluppo dell'organizzazione

Posto di lavoro

Comfort del lavoro nell'organizzazione

Stipendio

Vantaggi e bonus

Rapporti con Boss

Rapporti con i colleghi nell'organizzazione

Sicurezza del lavoro

Lavoro e vita privata$$
\text { ata }
$$

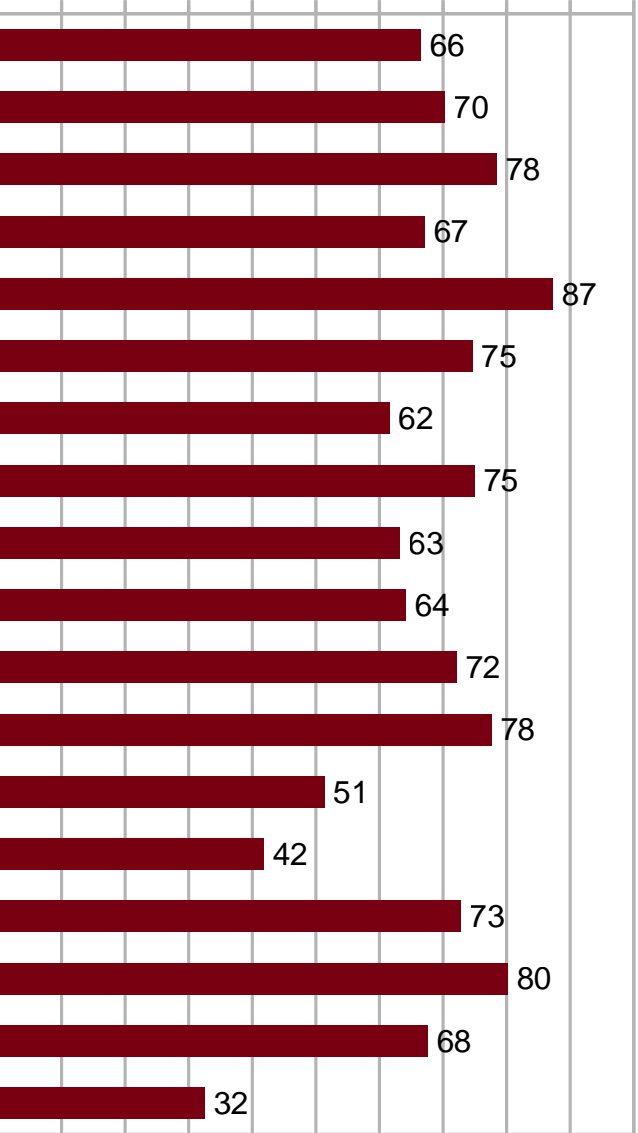

IL CONFRONTO DEI LIVELLI DI COMPETENZE CHIAVE TRA GL INTERVISTATI UE E NON UE

Nello studio sono stati registrati 1049 intervistati UE e 107 rispondenti non UE. Sebbene la sproporzione dei due gruppi sia visibile, si può presumere che il gruppo dell'UE a causa dell'ampio campione sia rappresentativo in modo convincente per i paesi dell'UE. Inoltre, va notato che il gruppo non UE soddisfa anche i requisiti minimi (usati nelle scienze sociali) di rappresentanza, dando così la possibilità di alcune conclusioni limitate ma convincenti.
Il punteggio medio generale del test AENI per i partecipanti dell'ue è stato del $78 \%$ e per i partecipanti non UE del $75 \%$. I risultati ottenuti mostrano quindi una leggera differenziazione dei punteggi, il che implica che gli educatori degli adulti dell'ue e i lavoratori del settore dell'istruzione degli adulti sono, ghi non UE. L'analisi più dettagliata incentrata sulle sottocategorie del test AENI ha rivelato la differenziazione dei punteggi nelle categorie comunicazione, digitale, imprenditore e apertura per le scienze. In tutte queste categorie, i partecipanti del'UE hanno ottent gon i partecipanti del ue hatenuto di gran lunga più soddisanti dei loro omolo-
Pertanto, gli educatori per adulti e i lavoratori delle ONG giudicano male i loro benefici materiali dal lavoro stesso. 
dei punteggi che vanno dall' $8 \%$ è stata osservata nell'apertura per la categoria scienza e cultura. Pertanto, si può presumere che gli intervistati dell'uE fossero più soddisfatti delle loro competenze in materia di comunicazione, digitale e soprattutto apertura per la scienza e la cultura rispetto ai loro omologhi non UE.
Figure 3. Punteggi della sottocategoria del test AENI Confronto dei partecipanti UE - non-UE

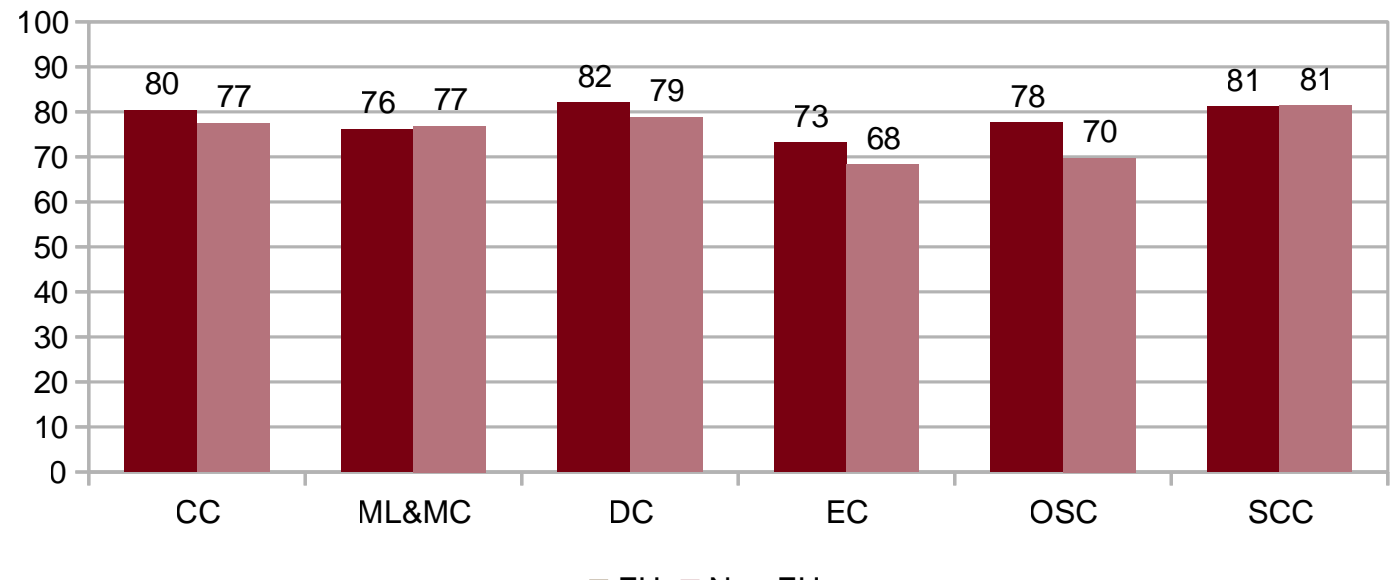

LA RACCOLTA DELLE ESIGENZE PER LA VALUTAZIONE DEL CAMBIAMENTO NELLE ORGANIZZAZIONI UE E NON UE

La valutazione del risultato dell'organizzazione ottenuto dal sondaggio ha rivelato che i punteggi medi generali per i nostri gruppi di intervistati confrontati erano del $69 \%$ per i partecipanti dell'Ue e de $67 \%$ per i partecipanti non UE. Pertanto, i partecipanti dell'UEvvalutano le loro orUn'analisi più dettagliata (Figura 4) delle singole categorie aveva rivelato una differenziazione più o meno visibile dei punteggi in tutte le categorie rispettate. Le differenze più visibili sono state rilevate nel raggiungimento del successo personale $(4 \%)$ e bonus (5\%), rapporto con il capo (4\%), ganizzazioni in modo leggermente più positivo rispetto alle loro controparti non UE. responsabilità $(6 \%)$, indipendenza $(6 \%)$ avanzamento $(5 \%)$, salario $(5 \%)$, benfici chevoli con i loro supervisori, dando loro di non interferire con la vita privata del dipendente. Le organizzazioni non UE hanno un impiego formale più stabile e cercando offerto ai propri dipendenti maggiori possibilità di raggiungere il successo personale, creare più opzioni di avanzamento e proporre vantaggi e bonus piu interessanti.
Figure 4. Valutazione dell'organizzazione

Punteggi medi nelle categorie - Confronto UE e non UE

$\begin{array}{lllllllllll}0 & 10 & 20 & 30 & 40 & 50 & 60 & 70 & 80 & 90 & 100\end{array}$

Apprezzamento da parte del leader Raggiungimento degli obiettivi per l'organizzazione

Raggiungimento del successo personale

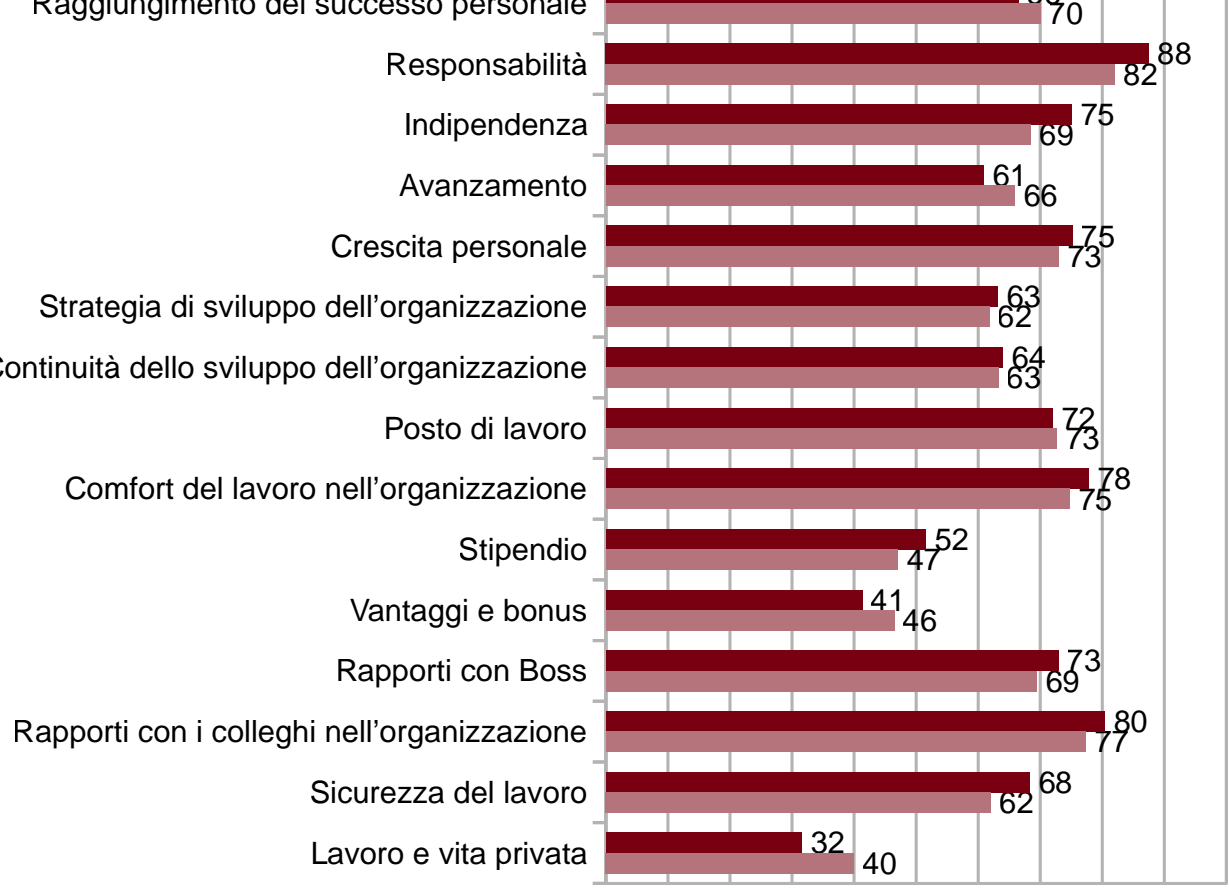

$\square$ Non-EU $\square$ EU

he gli intervistati dell'ue giudicano la loro organizzazione in modo più positivo in termini di responsabilità, indipendenza, stipendio, rapporti con il capo, sicurezza del lavoro e categorie di lavoro e vita privata. Gli intervistati non UE, d'altra parte, hanno elogiato le loro organizzazioni in aree come il raggiungimento del successo personale, l'avanzamento e le aree dei benefici e dei bonus.

Pertanto, si può concludere che le organizzazioni dell'Ue si pensava di dare ai propri dipendenti dipendenti un grado più elevato di responsabilità nel loro lavoro, una maggiore indipendenza nelle azioni e net prendere decisioni, modo più soddisfacente, creando relazioni più ami-
CONFRONTO DEI PUNTEGGI MEDI GENERALI DEI PAESI DEI PARTNER DEL PROGETTO NEL TEST DELLE COMPETENZE CHIAVE DELL'AENI

Nello studio sono stati registrati 201 partecipanti dal Portogallo, 195 partecipant dall'Italia, 135 partecipanti dalla Francia e 159 partecipanti dalla Polonia. Il confronto dei risultati medi rilevati nei singoli paesi aveva rivelato (Figura 5) Il punteggio più alto è stato notato in Italia (79\%), molto simile ma inferiore è stato notato in Francia e Portogallo (78\%) e il più basso è stato osservato in Polonia (76\%). Tutte le differenze rilevate possono essere classificate come molto elevate. 
Figure 5. Test AENI

Confronto del punteggio generale del paese

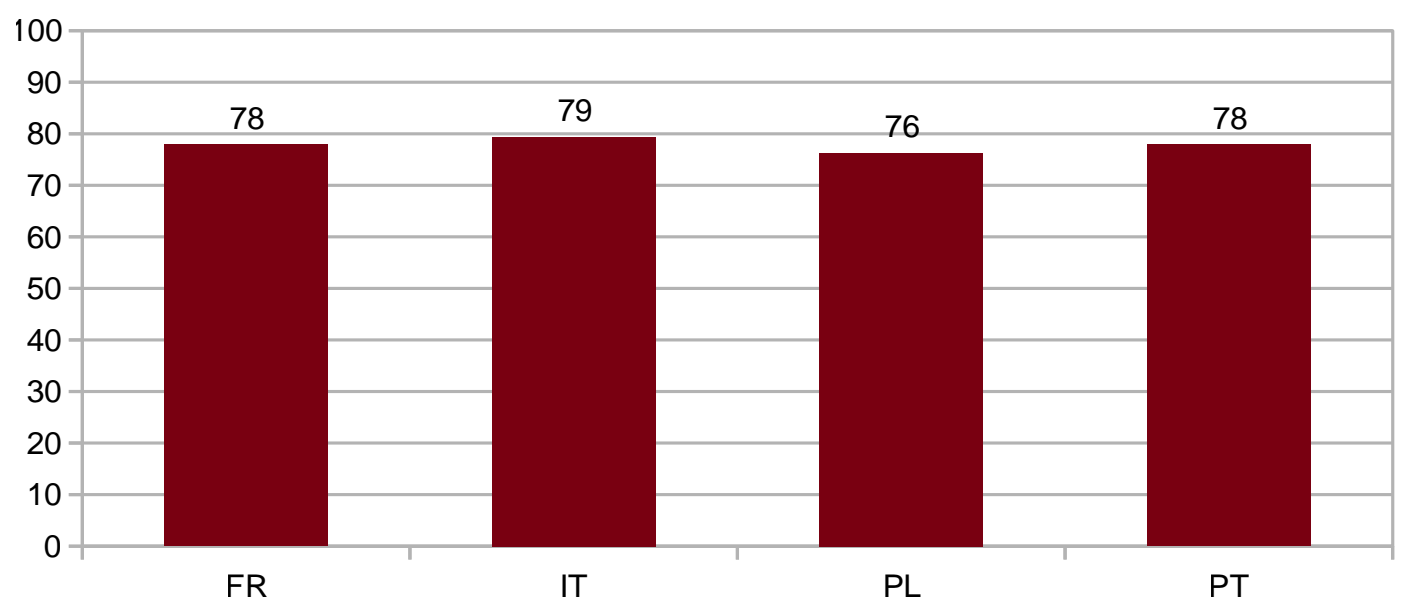

FR

PL

PT

\section{CONFRONTO DEI PUNTEGGI}

MEDI DEI PAESI DEI PARTNER DEL

PROGETTO NEL TEST AENI PER LE

RISPETTIVE COMPETENZE CHIAVE

Quando i punteggi generali sono stati suddivisi in sottocategorie, l'analisi compara-

tiva ha rivelato che la maggiore diversità

di punteggi è stata rilevata nelle aree mul-

tilingue, multiculturali e imprenditoria-

li. Nell'area multilingue e multiculturale,

il punteggio più alto è stato notato dai par-

tecipanti italiani $(77 \%)$ e quello pì̀ basso

è stato notato in Polonia (69\%). I punteg-

gi medi sono stati notati in Francia e Por-

togallo, ma erano più vicini ai punteggi ot-

tenuti dal campione italiano. Sulla base

dei risultati ottenuti si può presumere che

il campione polacco si distingue per i suo

punteggi negativi tra i gruppi confront

Pertanto, gli educatori polacchi per adulti e i lavoratori delle ONG possono avere esigenze di istruzione per quanto riguarda le questioni multilingue e multiculturali.

La seconda diversità più visibile nei punteggi è stata rilevata nell'area imprenditoriale, gli italiani (76\%).Il punteggio più basso è stato notato ancora una volta in Polonia ( $70 \%$ ). Un punteggio inferiore rispetto al campione italiano è stato notato anche dal campione francese $(72 \%)$. Sebbene i punteggi rilevati dai campioni polacchi e francesi possano essere classificati come buoni, mostrano chiaramente che ci sono alcune aree di esigenze educative riguardo alle competenze degli imprenditori che presuppongono un miglioramento da parte degli intervistati. Un punteggio inferiore è stato ottenuto dagli intervistati portoghesi $(75 \%)$ nell'apertura alla sottocategoria di scienza e cultura. (76\%)
Figure 6. Sottocategorie di test AENI

Confronto del punteggio medio del paese

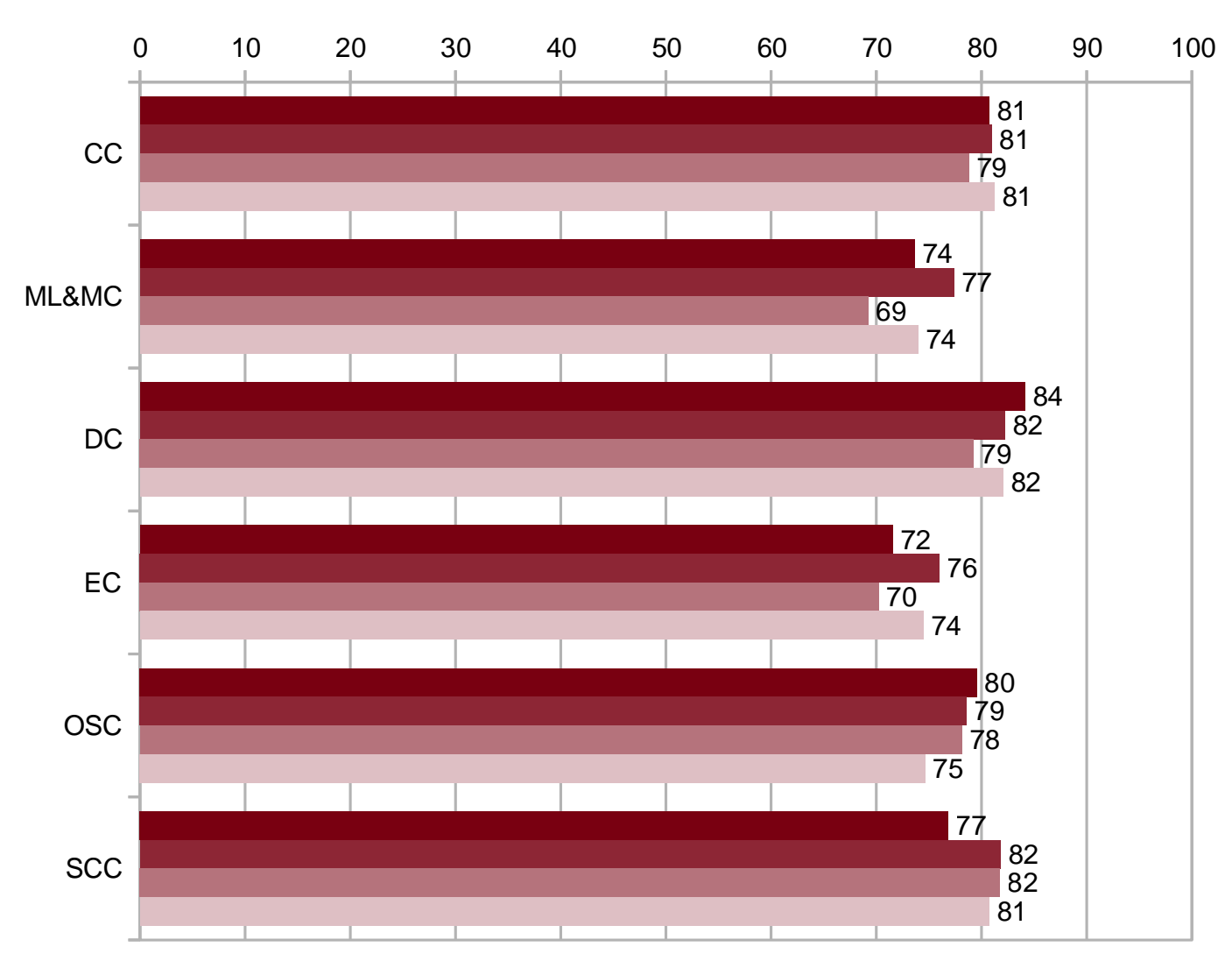

PT $\backsim \mathrm{PL} \backsim \mathrm{IT} \backsim \mathrm{FR}$

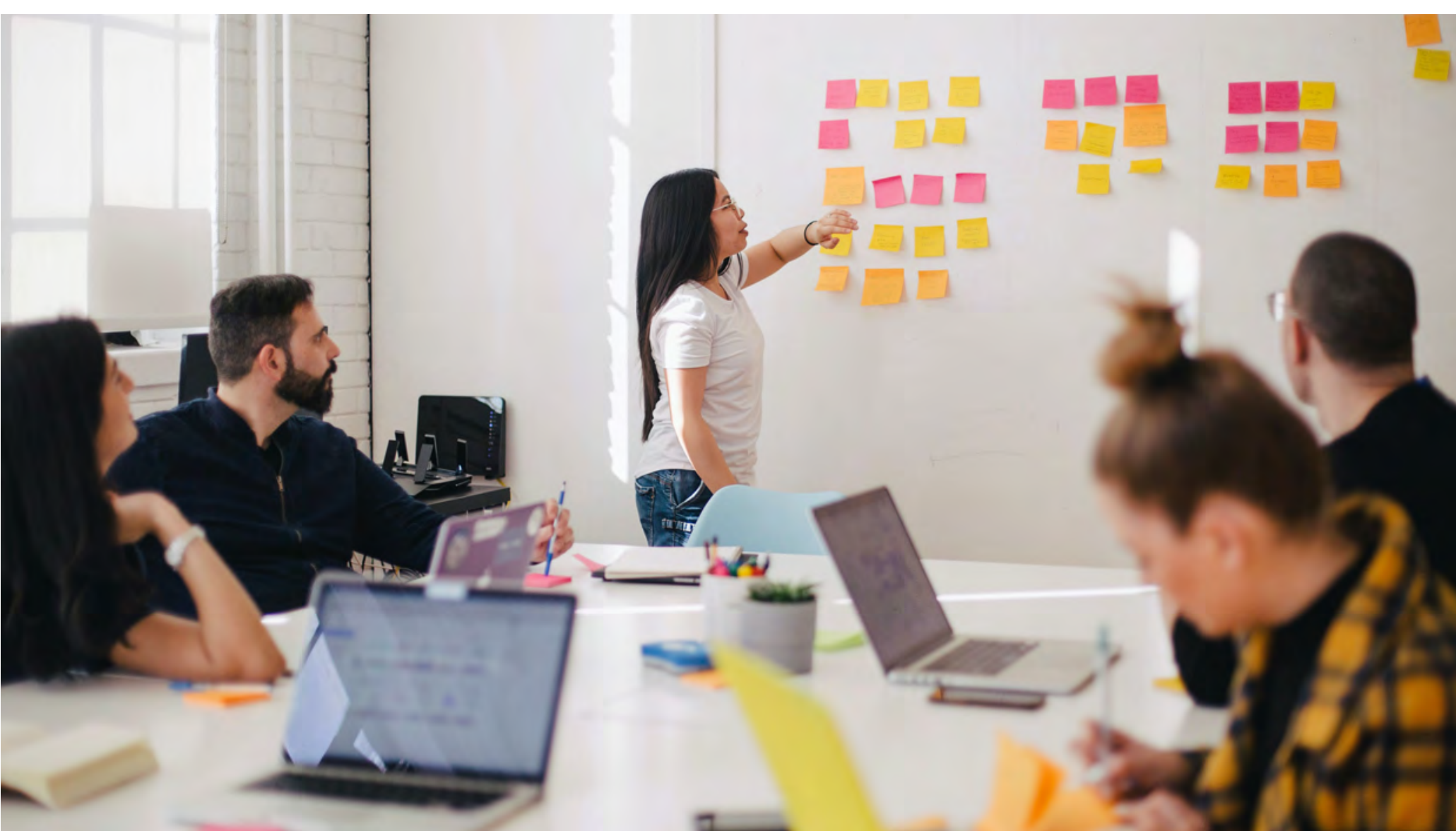




\section{VALUTAZIONE DELLE ESIGENZE DI CAMBIAMENTO NELLE}

ORGANIZZAZIONI

I punteggi relativi all'indagine sulla valutazione delle organizzazioni hanno rivelato che il punteggio medio più alto delle proprie organizzazioni è stato rilevato in Francia $(71 \%)$, leggermente inferiore in Portogallo $(69 \%)$ e notevolmente inferiore in Italia e Polonia (67\%). Tutti i punteggi delle note possono essere classificati come buoni.

Figure 7. Valutazione dell'organizzazione

Punteggio medio generale - Confronto tra paes

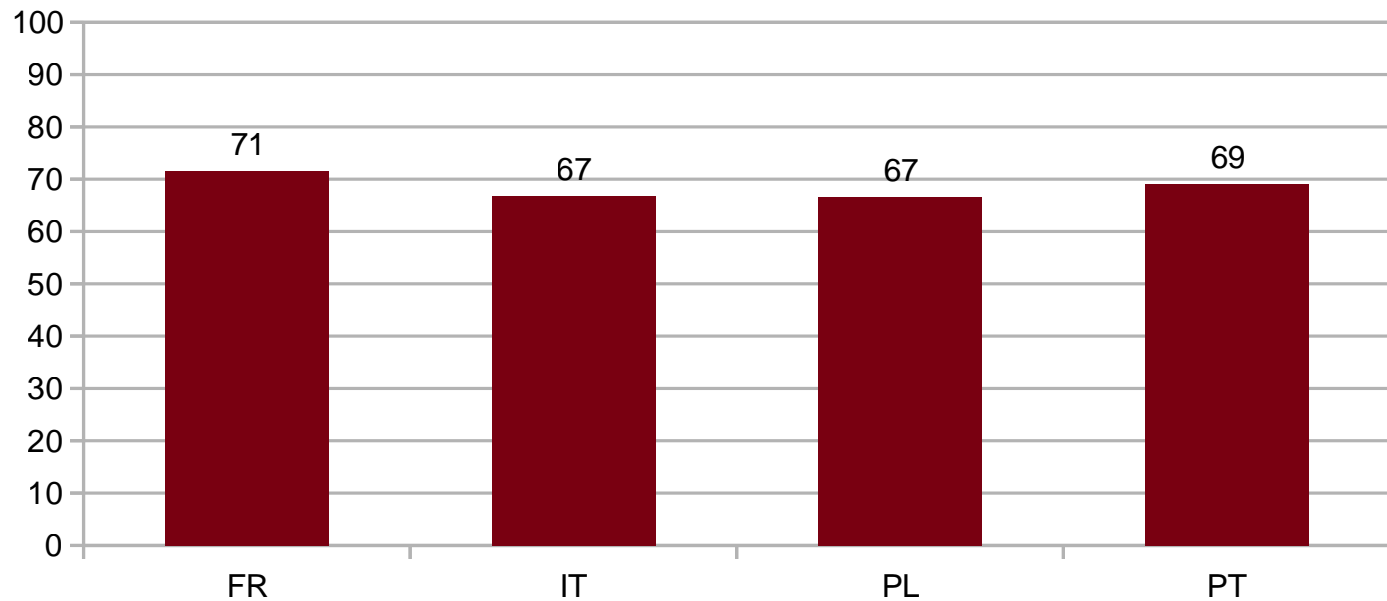

Quando divisi in categorie (Figura 8), i risultati hanno rivelato un'elevata varietà di punteggi in numerose aree. Il maggiore apprez zamento del datore di lavoro è stato rilevato in Francia $(73 \%)$ e il più basso in Polonia (58\%). L'apprezzamento del leader è stato visibilmente più elevato in Francia (75\%) rispetto a qualsiasi altro paese. Il raggiungimento degli obiettivi nell'organizzazione è stato più alto in Italia e Polonia (80\%). Il raggiungimento del successo personale è stato più elevato in Francia $(72 \%)$. Il maggio nel campione polacco (91\%). Il livell dipendenza è stato il più alto nel campione francese $(78 \%)$. Il livello di avanzamento è stato più alto nel campione francese $65 \%)$. Le maggiori possibilità di sviluppo personale sono state rilevate nel campione polacco (79\%). La strategia di sviluppo dell'organizzazione è stata ugualmente la più alta nel campione polacco $(65 \%)$ e in

Figure 8. Valutazione delle categorie organizzative Confronto del punteggio medio per paese

$\begin{array}{llllllllll}0 & 10 & 20 & 30 & 40 & 50 & 60 & 70 & 80 & 90\end{array}$

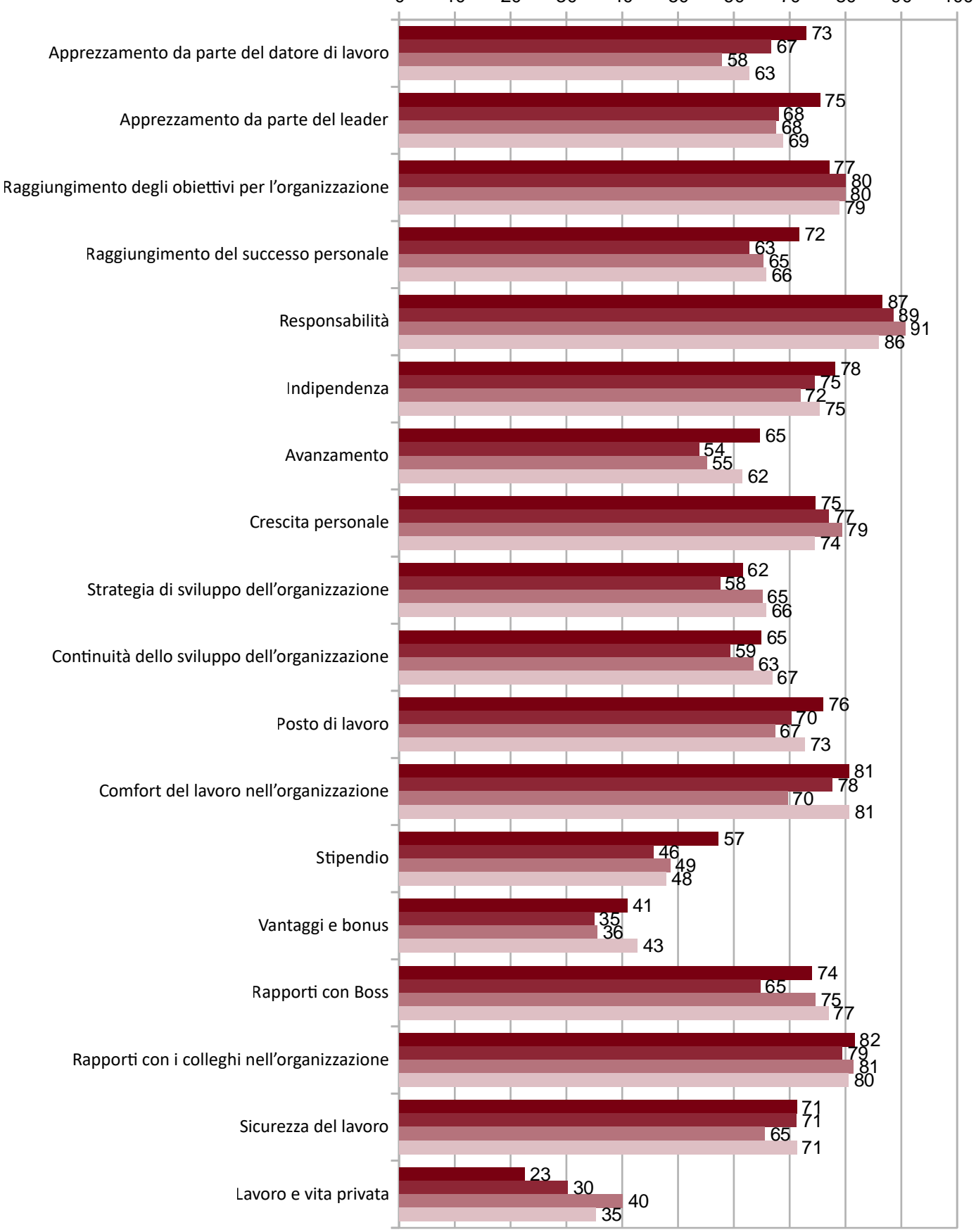

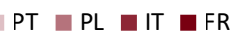

Portogallo (66\%). La continuità dello sviluppo è stata massima in Portogallo (67\%). Il posto di lavoro ha ottenuto il punteggio più alto in Francia (76\%). Il comfort di lavoro è stato altrettanto apprezzato in Francia e Portogallo ( $81 \%$ ). Lo stipendio è stato notato più alto in Francia (57\%) e il punteggio può essere qualificato come basso. I punteggi relativi a vantaggi e bonus possono essere classificati come negativi in tutti i paesi e vanno dal $35 \%$ al $43 \%$. Il rapporto con il capo è meglio visto nel campione del Portogallo (77\%). Il rapporto con i colleghi è valutato in modo molto equo in tutti i paesi e varia dal $79 \%$ all' $82 \%$. La sicurezza del lavoro è sorprendentemente la stessa in Francia, Italia e Portogallo con un risultato notevolmente inferiore della Polonia $(65 \%)$. L'impatto negativo più elevato de lavoro sulla vita personale è stato rilevato in Polonia (40\%), l'impatto più basso è stato registrato in Francia (23\%). 
PROFILO DELLE ORGANIZZAZIONI

PER L'EDUCAZIONE DEGLI ADULTI NEI

RISPETTIVI PAESI

Figure 9. Valutazione dell'organizzazione

Confronto dei punteggi inbase al tipo diorganizzazioni

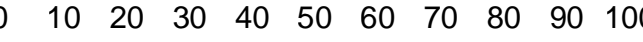

Apprezzamento da parte del datore di lavoro

Apprezzamento da parte del leader

Raggiungimento degli obiettivi per I'organizzazione

Raggiungimento del successo personale

$$
\text { Responsabilità }
$$

Indipendenza

Avanzamento

Crescita personale

Strategia di sviluppo dell'organizzazion

Continuità dello sviluppo dell'organizzazione

Posto di lavoro

Comfort del lavoro nell'organizzazione

Stipendio

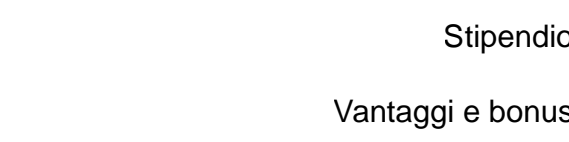

Rapporti con Boss

Rapporti con i colleghi nell'organizzazione

Sicurezza del lavoro

Lavoro e vita privata

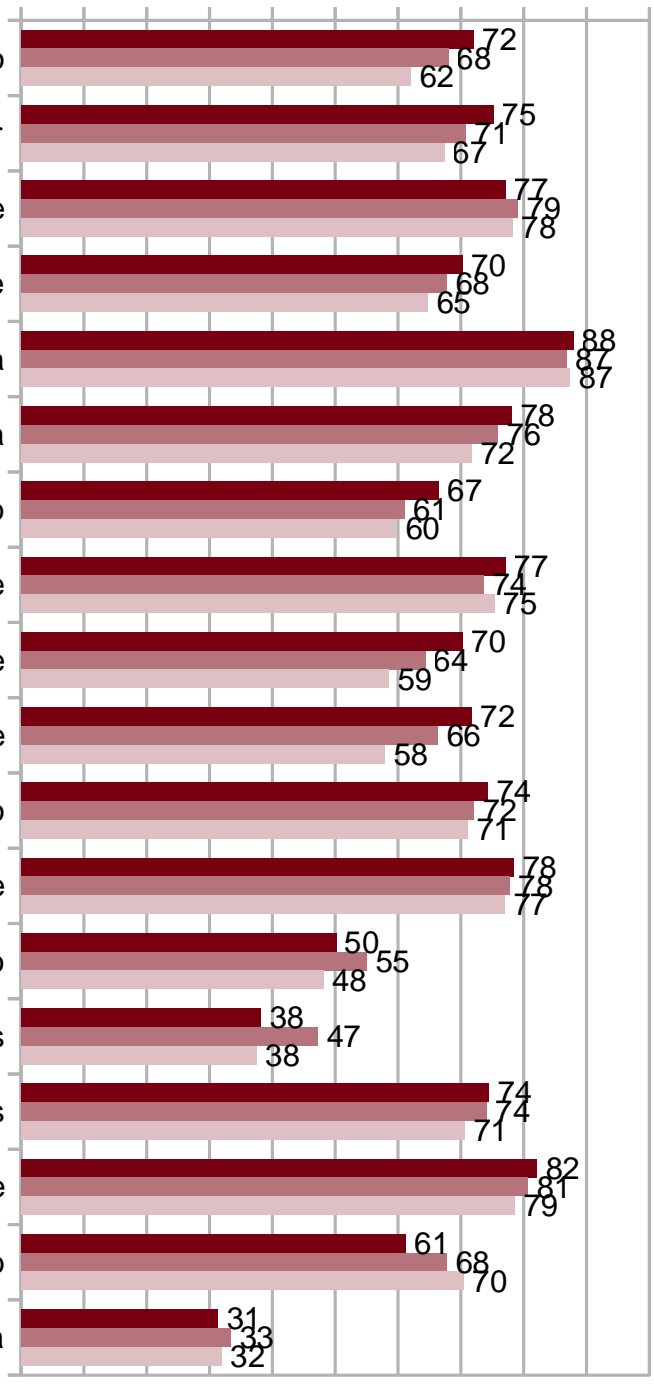

Statale $\mid$ Privata $=$ ONG
Sulla base dei dati raccolti, è possibile trarre conclusioni preliminari sui tipi di istituti di istruzione per adulti nei paesi dei partner del progetto.

Gli intervistati in Polonia hanno identificato ugualmente diversi tipi di organizzazioni (39 private, 32 statali e 33 ONG).

In Francia e in Italia, le organizzazioni per l'educazione degli adulti sono state identificate principalmente nel settore privato. In Francia, In Francia, gi in cato 98 organizzazioni private, 39 statal e 36 ONG, mentre in Italia erano 51 organizzazioni private, 44 statali e 38 ONG.

In Portogallo, c'era una sproporzione tra il numero di organizzazioni private (54) e ONG (50) e statali (34).

Questo confronto indica che il settore dell'educazione degli adulti delle ONG era il meno rappresentato (con il Portogallo un'eccezione), mentre il settore privato era il più rappresenato. La maggiore sproporzione tra i tipi di organizzazioni valutate si è verificata in Francia, mentre la più piccola in Polonia

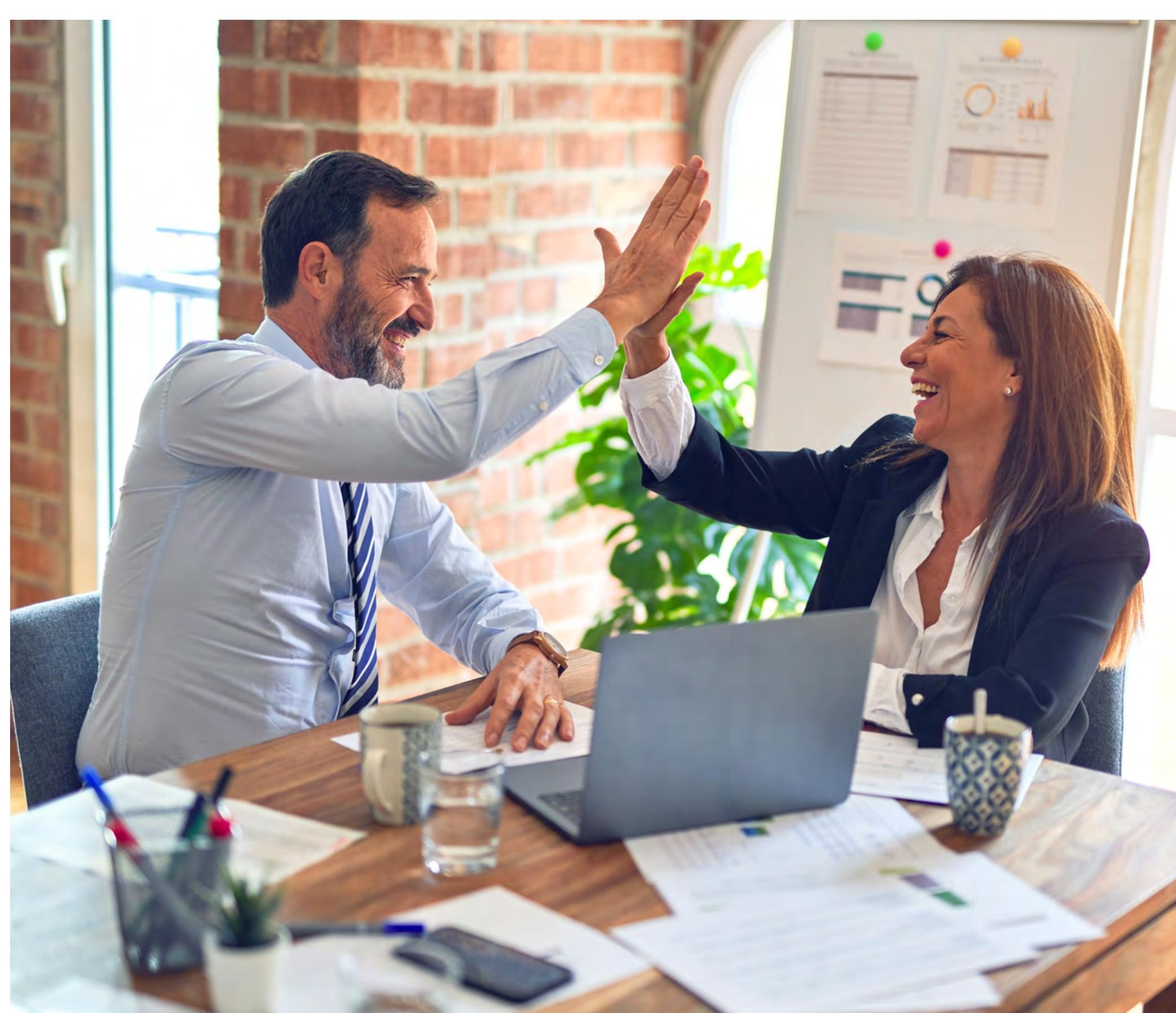


Fugure 10. Tipo di organizzazione valutata dagli intervistati

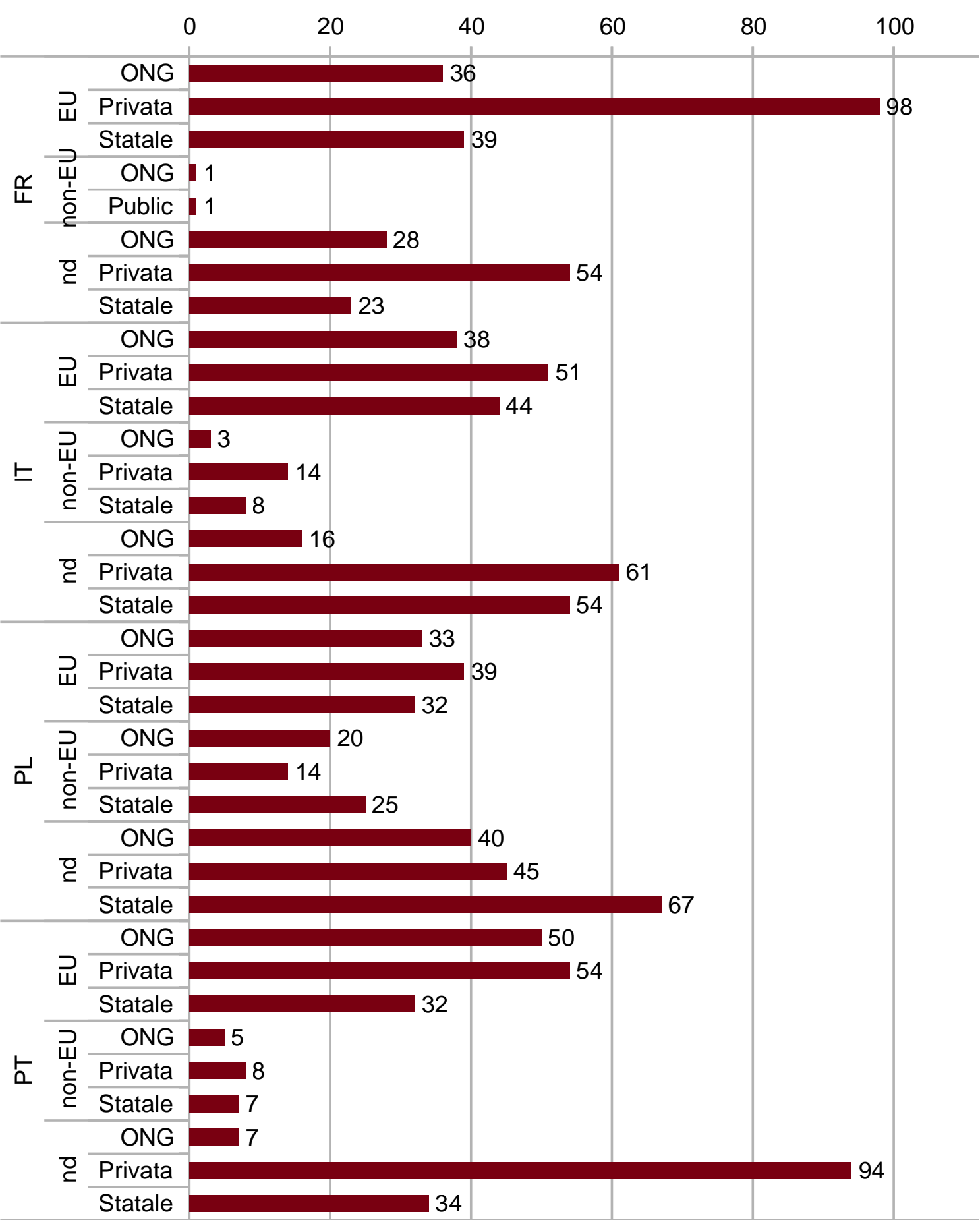

Il confronto della valutazione dei rispettivi tipi di organizzazione per quanto riguarda la loro necessità di cambiamento mostra che nella maggior parte dei casi le organizzazioni non governative sono valutate meglio di altri tipi di organizzazioni. a più forte esigenza di cambiamento è indicata dagli intervistati nei confro istituzioni statali. Queste tendenze diffe- riscono quando si tratta di indicatori puramente economici. Le organizzazioni non governative sono valutate come le peggiori per quanto riguarda stipendio, bonus e sicurezza del lavoro. Ciò indica maggiori esigenze di cambiamento in questo senso nelle ONG, ad es. sotto forma di aiuto finanziario o maggiore sicurezza del lavoro. 
3. Sommario

\section{SOMMARIO}

wes? 
Il progetto di ricerca era finalizzato alla diagnosi dei bisogni educativi e alla valutazione quantitativa delle organizzazioni da parte $\mathrm{d}$ educatori per adulti e lavoratori delle ONC. L'analisi comprendeva tre livelli di analisi 1) punteggi generali ottenuti dai partecipanti, 2) punteggi generali ottenuti da cittadin UE e non UE e 3) punteggi generali ottenuti dai partecipanti che rappresentavano le nazionalità dei partner del progetto.

Al primo livello di analisi - generale - è stato rilevato che i bisogni educativi più bassi sono collegati alle competenze digital $(82 \%)$ e il più alto era connesso alle competenze dell'imprenditore (73\%). La valutazione dell'organizzazione ha mostrato ch i partecipanti erano molto soddisfatti delle responsabilità che avevano $(87 \%)$ e la soddisfazione più bassa era con benefici e bonus $(42 \%)$ inclusa la bassa soddisfazion per lo stipendio $(51 \%)$

Al secondo livello di analisi, incentrato su confronto tra cittadini UE e non UE, è stato rilevato che nella maggior parte dei bisogn educativi il livello di soddisfazione dei partecipanti UE era notevolmente più alto. Un'eccezione è stata osservata nelle competenz multilingue e multiculturali che si sono rivelate $+1 \%$ a favore dei cittadini non UE. Valutazione dell'organizzazione indicata dall maggioranza dei punteggi, maggiore apprezzamento dell'organizzazione da parte dei cittadini UE con notevole eccezione nel raggiungimento del successo personale $(+4 \%)$ aree benefici e bonus $(+6 \%)$ a favore delle organizzazioni extracomunitarie.

Il terzo livello di analisi, incentrato sui partecipanti che rappresentano le nazionalità de partner del progetto, ha rivelato che i bisogni educativi sono meglio soddisfatti in Francia $(71 \%)$. Punteggi notevolmente inferior sono stati notati ugualmente in Italia e Polonia $(67 \%)$. Le sottocategorie avevano rive- lato che: in Francia, i bisogni maggiori sono nelle competenze degli imprenditori; in Italia e Polonia i bisogni più elevati sono l'area multilingue e multiculturale e le competenze imprenditoriali; in Portogallo, le esigenze più elevate riguardano l'area multilingue e multiculturale, le competenze imprenditoriali e l'apertura per la scienza e la cultura.

Per quanto riguarda la valutazione dell'organizzazione, i punteggi variavano così tanprese in considerazione solo valutazioni significative; di conseguenza, le sproporzioni significative sono state riscontrate nelle seguenti aree:

1) apprezzamento da parte del datore di lavoro,

2) apprezzamento da parte del leader,

3) raggiungimento del successo personale 4) avanzamento,

5) strategia di sviluppo dell’organizzazione 6) continuità di sviluppo dell'organizzazione

7) condizioni di lavoro,

8) comfort di lavoro,

9) condizioni salariali,

10) benefici e bonus,

11) rapporti con il capo,

12 ) sicurezza del lavoro

13) impatto del lavoro sulla vita personale.

I risultati mostrano chiaramente che i livelli generali degli educatori per adulti e dei bisogni educativi degli educatori degli adulti e la valutazione delle organizzazioni sono soddisfacenti. Eppure, c’è ancora spazio per miglioramenti e quelli si trovano in generale nelle aree di competenza multilingue e multiculturale e nelle competenze degli imprenditori. Pertanto, tutti i futuri progetti educativi volti al miglioramento delle esigenze degli educatori degli adulti e dei lavoratori delle ONG dovrebbero concentrarsi su quelle aree. I maggiori inconvenienti delle organizzazioni che impiegano educato voratori del settore dell'istruzione degli adul- ti si trovano nei salari e nei bonus finanziari che vengono diagnosticati a un livello critico e sono una parte importante del proprio senso materiale di sicurezza e possono essere un fattore importante per attrarre persone talento per questo tipo di attività. Inoltre, si richiama l'attenzione sul fatto che il sottofinanziamento da parte delle istituzioni soddisfa le elevate competenze degli educatori.

I cittadini dei paesi dell'UE hanno generalmente bisogni molto soddisfatti rispetto cittadini dei paesi non UE e inoltre lodano

la loro organizzazione più in alto. Tuttavia hanno un livello inferiore di raggiungimento del successo personale, il che implica che gli educatori per adulti dell'uE e i lavoratori delle ONG non collegano la loro attività così fortemente con il successo personale che può avere premesse culturali o economiche.

Infine, se si prendono in considerazione solo i partecipanti che rappresentano i paesi coinvolti nel progetto, c'è una premessa significativa che indica h necessità di supportare i cambiamenti nelle seguenti aree:

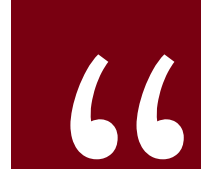

apprezzamento dell'area datoriale, soprattutto per quanto riguarda la Polonia;

apprezzamento per area leader in tutti i paesi diagnosticati positivamente esclusa la Francia;

च raggiungimento dell'area di successo personale, soprattutto per quanto riguarda l'Italia;

च area di avanzamento soprattutto in Italia e Polonia;

च strategia di sviluppo dell'area organizzativa, soprattutto in Italia;

च continuità di sviluppo dell'area organizzativa, soprattutto in Italia;

area condizioni di lavoro, soprattutto in Polonia;

च comodità di lavoro nell’area dell’organizzazione, specialmente in Polonia;

च area delle condizioni salariali in tutti i paesi diagnosticati;

area benefici e bonus che che sono stati valutati male in tutti i paesi;

घapporti con l'area boss, soprattutto in Italia;

च sicurezza del lavoro, soprattutto in Polonia;

■ limpatto del lavoro sulla vita privata, soprattutto in Polonia. 
Il rapporto è stato valutato da un valutatore indipendente, Tijana Milenkovic Jankovic (Regno Unito). Ha lavorato su diversi progetti internazionali nel campo dell'istruzione (continua e per adulti) per più di 20 anni. Questa esperienza lavorativa le ha fornito l'opportunità di approfondire e comprendere il funzionamento delle istituzioni e delle organizzazioni educative

\section{Le sue principali conclusioni sono:}

1. AENI fornisce una solida visione generale dei bisogni degli educatori degli adulti. Anche se i risultati generali del test AEN mostrano risultati principalmente elevati in tutti i paesi partner, i risultati si basano sull'autovalutazione. [...] I risultati ricevuti possono essere un'indicazione per le parti interessate nei paesi partner sulle competenze più necssaie per gli educatori degli tenze pau necessarie per gli educatori deg adulti, che possono servire per pianificar come migliorarle all'interno dell'istruzion iniziale, nonché nei corsi di sviluppo professionale continuo (CPD) per gli educatori degli adulti, ma anche per supportare il processo di validazione delle competenze acquisite in contesti diversi (Risoluzione Consiglio, 2011). Consiglio, 2011). [...] A livello generale, la maggioranza dei punteggi è risultata alta. in vari settori e in tutte le fasi del ciclo educativo. Ha maturato esperienza nell'area della valutazione come consulente indipendente nell'ambito del progetto di valutazione del programma di formazione professionale dell'UNHCR in Serbia e come a Lux-Development Freelance Regional / National Consultant in Montenegro.

Tuttavia, la particolare esigenza è identificata nelle competenze multilingue e multiculturali $(M L \& M C)$ e nelle competenze imprenditoriali $(C E)$. [...]

2. Sarebbe importante che le organizzazioni coinvolte nella ricerca ricevano i risultati dello studio e abbiano la possibilità di confrontare i risultati con i risultati generali a livello di paese, ma anche di confrontarli con altri paesi. I risultati possono servire a riflettere sui loro risultati e fare i piani d'azione per migliorare la situazione nell'organizzazione. [...]

3. Lo strumento quantitativo sviluppato per la valutazione delle organizzazioni ha fornito una valida base per la preparazione della ricerca qualitativa più approfondita. 
4. References

\section{REFERENCES}

whe 
Bacigalupo, M., Kampylis, P., Punie, Y., \& Van den Brande, G. (2016). EntreComp: The entrepreneurship competence framework. Luxembourg : Publication Office of the European Union, 10, 593884

Bassett-Jones, N., \& Lloyd, G. C. (2005),Does Herzberg's motivation theory have staying power? Journal of Management Development, Vol. 24 Iss 10 pp. 929-943, http:// dx.doi.org/10.1108/02621710510627064.

Buiskool, B. J., Broek, S. D., van Lakerveld, J.A Zarifis, G. K., \& Osborne, M. (2010). Key competences for adult learning professionals. Contribution to the development of a reference framework of key competences for adult learning professionals, 157

Burns, D. (1985). Intimate connections. New York: Signet (Penguin Books).

Caena, F. (2013). Supporting teacher competence development for better learning outcomes. Education \& Training, European Commission, p. 5-59.

Council of Europe. Council for Cultura Co-operation. Education Committee. Modern Languages Division. (2001). Common European Framework of Reference for Languages: learning, teaching, assessment. Cambridge University Press.

Council of the European Union. (2018). COUNCIL RECMMENDATIONS, 22 May 2018, with reference to key competences in the process of life-long learning. (2018/C 189/1). Official Journal of the European Union, pp. 1-13

Council Resolution on a renewed European agenda for adult learning $O J \mathrm{C}_{372}$ 20.12.2011, p. 1-6.

EU Science Hub - European Commission 2020. Entrecomp: The Entrepreneurship
Competence Framework. - EU Science Hub European Commission. [online] Available at: <https://ec.europa.eu/jrc/en/publication/eur-scientific-and-technical-researchreports/entrecomp-entrepreneurship-com[Accessed 15 November 2020].

Europa.eu. 2020. Common European Framework of Reference. Europass. [online] Available at: <https://europa.eu/europass/en/ common-european-framework-reference> [Accessed 15 November 2020].

Faure, E., Herrera, F., Kaddoura, A. R., Lopez, H., Petrovski, A. V., Rahnema, M., \& Ward, F. C. (1972). Learning to be: the world of education today and tomorrow. Paris: UNESCO.

Głomb, K. (2020). Edukacja dla Przemystu 4.o. Wyzwania dla Polski [Education for Indus-try 4.o A Challenge for Po land]. Warszawa: Agencja Rozwoju Przemystu.

Griffith, W. S. (1978). Educational Needs: Definition, Assessment, and Utilization. The School Review, 86(3), pp. 382-394.

Hakio, K., \& Mattelmäki, T. (2019). Future Skills of Design for Sustainability: An Awareness-Based Co-Creation Approach. Sustainability(11), pp. 1-24.

Herzberg, F., Mausner, B. and Snyderman, B. (1959), The Motivation to Work, Wiley, New York,NY

Hipkins, R. (2018). How the key competencies were developed: The evidence base. Wellington: New Zealand Council for Educational Research.

Kobylarek, A. (2009). Kompetencje komunikacyjne w systemie umiejetności nauctem of teachers' abilities]. In K. Błaszczyk,
M. Drzewowski, \& W. Maliszewski, Komunikacja spoteczna a zarządzanie we wspótczesnej szkole [Social communication and management in a modern school] (pp. 334342). Toruń: Adam Marszałek.

Martowska, K., \& Matczak, A. (2013). Pomiar kompetencji społecznych - prezentacja nowego narzędzia diagnostycznego [Measuring social skills - presentation of a new diagnostis tool]. Psychologia Jakości Życia [Psychology of the Quality of Life] (1) pp. 43-56.

Morris, E. (2001). Special Educational Needs. Code of Practice. London: Department for Education and Skills.

Nijssen, A., van Lakerveld, J. A., Buiskool, B. J. den Oudendammer, F., Broek, S. D., \& Hake, B. (2008). ALPINE - Adult Learning Profession in Europe: A Study of the Current Situation, Trends and Issues. Project Report. Research voor Beleid, Zoetermeer, Netherlands.

Padzik, K. (2016). Ocena zintegrowana Assessment i Development Center. Warszawa: Wolters Kluwer.

Paterson, R. (2000). The Assertiveness Workbook: How to Express Your Ideas and tionships. Oakland: New Harbinger.

Petrètiène, A., Daukšienė, J., \& Grašienė, J. (2020). Creativity and speciality language in the context of the development of key competences. Mokslas - Lietuvos ateitis(12), pp. 1-7.

Radovan, M. (2019). Cognitive and Metacognitive Aspects of Key Competency "Lear ning to Learn”. Pedagogika(1), pp. 28-41.

Stufflebeam, D. L., McCormick, C. H., Brinkerhoff, R. O. \& Nelson, C. O. (2012). Conducting Educational Needs Assessments. Springer.
Warzocha, T. (2016). Kompetencje komunikacyjne jako komponent kompetencji spolecznych nauczycieli akademickich - założenia do badan [Communication skills as a component of social skills for acad kacja - Technika - Informatyka [Education - Engineering - Information Technologies] (2), pp. 70-75. 
5. Annexes

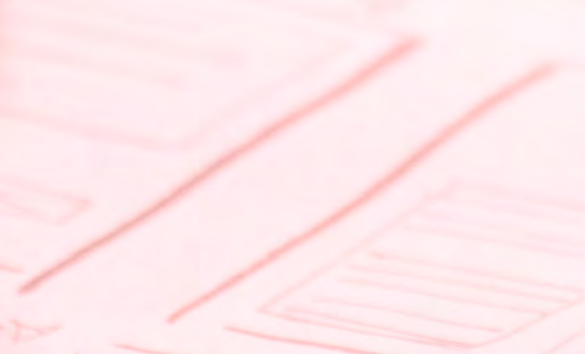


(Adults Education Needs Inventory)

Questionario per studiare i bisogni formativi nel campo delle competenze chiave degli adulti

Qui sotto cè una lista di 39 affermazioni. Per favore indicate quanto siete d'accordo con ogni affermazione su una scala da 1 a 5 ( 1 = decisa-

mente in disaccordo, $\mathbf{5}$ = decisamente d'accordo). Non ci sono risposte buone o cattive,

N.B.: Si prega di rispondere a tutte le domande

$1=$ decisamente in disaccordo $5=$ decisamente d'accord

1 CC Posso avere una conversazione in qualsiasi situazione

(ad es. durante un conflitto o in caso di opinioni divergenti)

CC Mi considero una persona in grado di mandare messaggi chiari e comprensibili

3 SCC Quando cerco informazioni cerco di usare varie risorse

$4 \mathrm{R}$ CC Molto spesso sono in situazioni in cui trovo difficile esprimere i miei pensieri

5 CC Di solito non ho problemi a capire il mio interlocutore

6 CC Mi piace discutere con varie persone

CC Mi sento a mio agio ad avere conversazioni con altre persone

CC Posso esprimere i miei pensieri in modo non conven-

zionale

9 MM Posso parlare e scrivere fluentemente in più di una lingua straniera

OSC Ritengo di non aver bisogno di sviluppare la mia conoscenza delle lingue straniere

MM Colgo diverse opportunità per l'apprendimento delle lingue straniere

12 MM Mi piace imparare nuove lingue

MM Uso una lingua straniera tutti i giorni (ad es. guardando

13 film, leggendo libri) 
OSC Ritengo che la matematica non aiuti a scoprire

la verità

OSC Vi sono dubbi giustificabili su alcune teorie, (ad es.

fluenza dell'uomo sul clima o l'uso della vaccinazione)

16 EC Ritengo che un'azione efficace richieda un piano chiaro

e inequivocabile

17R EC A volte agisco illogicamente

18 SCC Spesso uso varie fonti durante la pianificazione

19 EC In genere seguo un piano predeterminato

20 DC So usare le nuove tecnologie per una comunicazione

più efficace

21 DC Utilizzo varie innovazioni tecnologiche

22 DC Sento di essere abbastanza competente nella tecnologia

2 dell'informazione

DC Posso usare facilmente i dispositivi più comuni

(PC, smartphone, laptop, tablet, ecc.)

24 CC Posso lavorare in gruppo

25R CC Penso che sia difficile per me stabilire relazioni con altre persone

26R EC A volte sono difficile nei rapporti con altre persone

OSC È impossibile conciliare la politica di integrazione dell'UE con la peculiarità culturale

28 SCC Mi occupo di questioni sociali che sono importanti per me

29 SCC Sento di avere un effetto su ciò che mi circonda

30 CC Posso motivare gli altri a realizzare obiettivi comuni

31 CC Sono efficace nelle negoziazioni

$32 \mathrm{R}$ OSC Il mondo non offre molte possibilità per realizzare le mie idee

33R EC Non mi piace la fase di pianificazione

34R EC Ritengo che il controllo e il monitoraggio del lavoro siano generalmente inutili

EC A volte percepisco la mia cattiva gestione durante

l'esecuzione di un compito

36R CC Raramente do feedback ai miei colleghi

MM Sono interessato a varie culture

OSC Non sono in grado di esprimermi in nessuna forma d'arte

OSC Per me la cultura contemporanea non ha valore

CC Competenze di comunicazione (max. 60 pts.): 1, 2, 4R, 5, 6, 7, 8, 24, 25R, 30, 31, 36R

MM Multilinguismo e multiculturalismo (max. 25 pts.): 9, 11, 12,13, 37

DC Competenze digital (max. 20 pts.): 20, 21, 22, 23

EC Competenze imprenditoriali (max. 35 pts.): 16, 17R, 19, 26R, 33R, $34 \mathrm{R}, 35 \mathrm{R}$

OSC Apertura alla scienza e alla cultura (max. 35 pts.): 10R, 14R, 15R, 27R, 32R, 38R, 39R

SCC Competenze sociali e civili (max. 20 pts.): 3, 18, 28, 29

R Punteggio inverso

\section{NTERPRETAZIONE DEI RISULTATI}

La standardizzazione della ricerca è attualmente in corso su un gruppo che rappresenta educatori di vari paesi europei. In questa fase gli autori propongono l'uso di norme fase gli autori propongono l'uso di norme
provvisorie per aiutare nella conversione dei provvisorie per aiutare nella conversione dei
dati grezzi. A seconda del numero di punti ottenuti in ogni particolare classe, è possibile assegnare i risultati a uno dei tre gruppi: alto, medio e basso. Risultati bassi indicano un bass livello dicompenza in un particoenza in un particoare educatore e quindi una maggiore necessità di migliorare quella competenza.

\section{Interpretazione dei risultati}

\begin{tabular}{|lccc} 
& basso & medio & alto \\
\hline CC & $12-13$ & $32-41$ & $42-60$ \\
\hline MM & $5-13$ & $14-17$ & $18-25$ \\
\hline DC & $4-10$ & $11-14$ & $15-20$ \\
\hline EC & $7-18$ & $19-24$ & $25-35$ \\
\hline OSC & $7-18$ & $19-24$ & $25-35$ \\
\hline SCC & $4-10$ & $11-14$ & $15-20$ \\
\hline
\end{tabular}




\section{VALUTAZIONE DELL'ORGANIZZAZIONE}

Questionario che esamina la necessità di cambiamenti nelle attività dell’organizzazione

Di seguito sono riportate 18 dichiarazion relative alla tua opinione sul luogo di lavo-

ro. Indica in che misura sei d'accordo con

ciascuna affermazione su una scala da 1 a 5 $(\mathbf{1}=$ decisamente non d'accordo, $5=$ decisamente d'accordo)

N.B.: Si prega di rispondere a OGNI domanda

$1=$ decisamente in disaccordo, 5 = decisamente d'accord

40 Mi sento apprezzato dal mio datore di lavoro

41 Mi sento apprezzato dal mio capo (manager, leader, ecc)

42 Raggiungo completamente gli obiettivi stabiliti dal mio datore di lavoro

3 Il mio posto di lavoro mi permette di raggiungere il successo

44 Mi sento responsabile della mia posizione e dei compiti che mi vengono assegnati

45 Mi sento indipendente nello svolgimento dei compiti che mi sono stati assegnati

46 Il mio datore di lavoro mi offre l'opportunità di avanzamento di carrier

Il mio lavoro attuale influenza il mio sviluppo personale

$47 \quad$ (espandere la mia conoscenza, imparare qualcosa di nuovo)

48 Il mio datore di lavoro ha una direzione precisa nello sviluppo dell'organizzazione

49 Il mio datore di lavoro non riposa sugli allori e sviluppa continuamente l'organizzazione

50 La mia posizione a lavoro è adatta alle mie funzioni

51 Mi sento a mio agio nell'esercizio delle mie funzioni sul lavoro

52 Guadagno abbastanza nella mia organizzazione

53 L'organizzazione mi offre vantaggi e bonus interessanti

54 La mia relazione diretta con il mio capo è soddisfacente 
56 La mia organizzazione mi garantisce la sicurezza del lavoro

$57 \quad$ Il mio lavoro ha un effetto negativo sulla mia vita privata

Per favore indicate

58. Sesso $\square$ M $\quad \square$ F

59. Età .............

60. Tipo di organizzazione $\square$ statale

$\square$ Privata

$\square$ ONG

61. Posizione (contrassegnare solo UNA con una $\mathrm{X}$ )

\begin{tabular}{l|l}
\hline $\begin{array}{l}\text { proprietario / direttore } \\
\text { dell'organizzazione }\end{array}$ & \\
\hline manager intermedio & \\
\hline dipendente & \\
\hline volontario & \\
\hline
\end{tabular}

\section{INTERPRETAZIONE DEI RISULTATI}

I punteggi sono rappresentati in valori $\%$. Ogni elemento può avere un punteggio minimo dello $\% \%$ e un punteggio massimo del $100 \%$. Più alto è il punteggio che l'individuo ottiene, migliore è la sua opinione della sua organizzazione. L'unica eccezione è l'ultima categoria focalizzata sul lavoro e sulla vita privata privata, in questa categoria minore è il punteggio ottenuto più debole è l'influenza de lavoro sulla propria vita privata, quindi migliore è il punteggio.

Si raccomanda di interpretare i punteggi ottenuti riguardo alla valutazione dell'organizzazione secondo la classificazione proposta di seguito:

0-20\% - molto bassa- pessima opinione sull’organizzazione / datore di lavoro

21-40\% - bassa - cattiva opinione sullorganizzazione / datore di lavoro

41-60\% - media - opinione neutra sull'organizzazione / datore di lavoro

61-80\% - alto - buona opinione sull'organizzazione / datore di lavoro

81-100\% - molto alto- ottima opinione sull'organizzazione / datore di lavoro

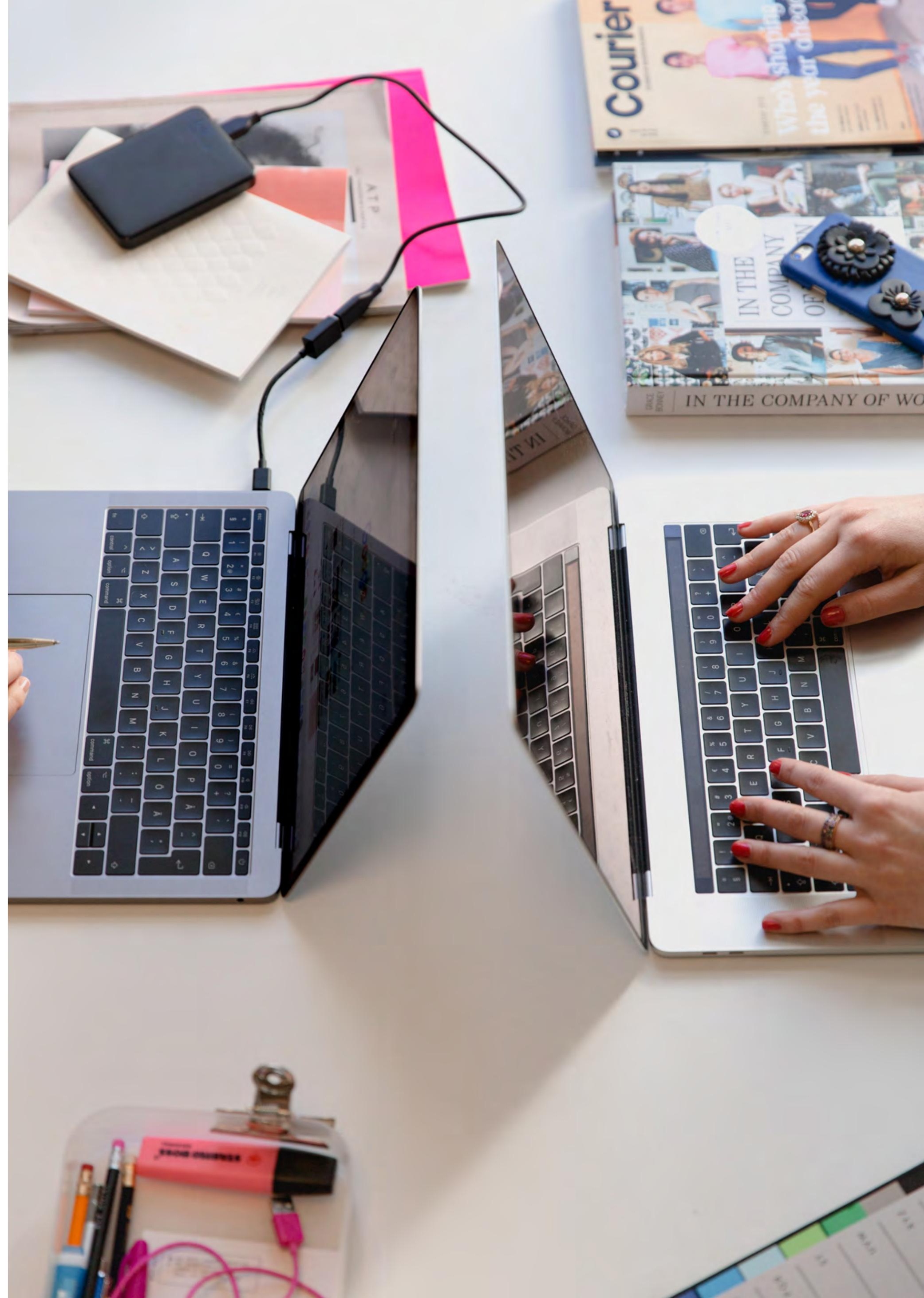



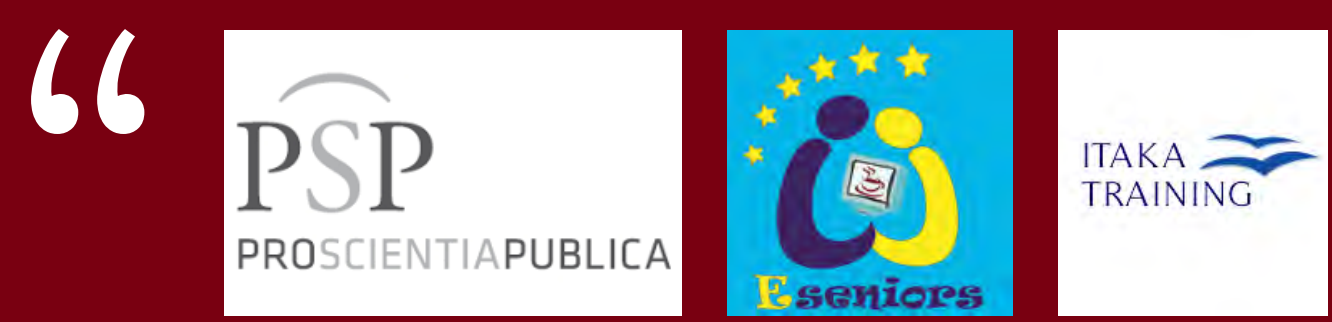

ISBN 978-83-953451-7-3

9

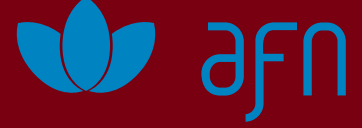

